diamond layer, which, thanks to its high thermal conductivity, provided efficient heat dissipation. When optically pumping the device at a wavelength of $980 \mathrm{~nm}$, the researchers obtained an output power of up to $100 \mu \mathrm{W}$ and a side-mode suppression of more than $30 \mathrm{~dB}$. They say that improving the bonding between the active multiquantum well layer and the heat sink layers below might increase the lifetime of the device.

\section{ULTRAFAST PHOTONICS}

\section{Ultralow timing jitter}

Opt. Lett. 36, 4443-4445 (2011)

Femtosecond mode-locked erbium-doped fibre (Er-fibre) lasers operating in the telecommunications wavelength range of 1.5-1.6 $\mu \mathrm{m}$ are a valuable light source for optical communications, such as long-distance clock distribution via fibreoptic links. So far, the best timing jitter performance demonstrated for free-running Er-fibre lasers is 2.6 fs. Tae Keun Kim and co-workers from the Korea Advanced Institute of Science and Technology have now demonstrated optical pulse trains with timing jitters of less than 100 as from two nearly identical $78 \mathrm{MHz}$ free-running mode-locked Er-fibre lasers. Both lasers had a central wavelength of $1,582 \mathrm{~nm}$ and a pulse width of $\sim 60 \mathrm{fs}$. One of the lasers contained a sigma cavity with a piezoelectric transducer mounted mirror in its free-space section to enable repetition-rate mode-locking between the two lasers. The net cavity dispersion was set to $-0.002 \pm 0.001 \mathrm{ps}^{2}$ at the central wavelength by balancing the positive dispersion of the Er-fibre and the negative dispersion of standard single-mode fibre. At high offset frequencies of above $8 \mathrm{MHz}$, the timing jitter spectral density was around $10^{-12} \mathrm{fs}^{2}$. The integrated root-meansquare timing jitter for offset frequencies of $10 \mathrm{kHz}$ to $38.8 \mathrm{MHz}$ was $70 \mathrm{as,} \mathrm{which} \mathrm{is,}$ according to the team, the lowest timing jitter ever achieved for free-running modelocked fibre lasers.

\section{TOPOLOGICAL INSULATORS \\ Light controls currents}

Nature Nanotechnol. http://dx.doi.org/10.1038/ nnano.2011.214 (2011)

James McIver, David Hsieh and co-workers from the Massachusetts Institute of Technology and Harvard University in the USA have experimentally observed optically driven currents in a topological insulator. Theory predicts that circularly polarized light should be able to drive spin-polarized net electrical current because the light would induce interband transitions whose probability depends on the surface-state spin orientation, which in turn depends on the momentum (and hence the polarization). In their experiment, the researchers used $795 \mathrm{~nm}$ light and $\mathrm{Bi}_{2} \mathrm{Se}_{3}$, which is a topological insulator because the currents are allowed only near the surface owing to spin-degeneracy of the bulk bands. One of the main challenges in their work was separating and eliminating surface and bulk currents generated by other effects, such as laser-induced thermoelectric currents. The researchers therefore experimented with heat gradients

\section{OPTOMECHANICS}

\section{Reciprocal action}

Coupling the motion of trapped atoms to the vibrations of a single mode of a mechanical oscillator is useful for a variety of reasons: it enables the oscillator's dissipation to be engineered and its motion to be read out, and eventually allows the exchange of quantum information between the two systems. Now, by coupling laser-cooled atoms to a micromechanical membrane, Stephan Camerer and colleagues from Germany and Switzerland have experimentally coupled membrane vibrations to ultracold atoms and the backaction of the atomic motion to the membrane. They have also demonstrated the ability to engineer the dissipation rate of the membrane. In their set-up, the beam from a grating-stabilized diode laser is sent through a vacuum chamber containing ultracold ${ }^{87} \mathrm{Rb}$ atoms and is partially reflected at the surface of an SiN membrane oscillator mounted in a separate vacuum chamber. The overlapped reflecting and incoming laser beams form a one-dimensional optical lattice with a beam waist of $350 \pm 30 \mu \mathrm{m}$ at the position of the atoms, which establishes a long-distance coupling between the ultracold atoms and the membrane. When the trap frequency of the atoms in the lattice matches the eigenfrequency of the membrane, the coupling leads to resonant energy transfer between the two systems. The researchers obtained remarkable agreement between theory and experimental results. Their findings highlight the potential use of atoms for cooling membranes down to the quantum ground state. between the device contacts with swept laser spots, and also used low laser intensities to minimize sample heating. After separating out any interfering effects, the team confirmed not only that the circularly polarized light generated a photocurrent, but also that the direction of the photocurrent can be controlled by the direction of the light momentum, as predicted.

\section{COLLOIDAL NANOCRYSTALS}

\section{Excited states investigated}

Nanotechnol. 23, 015201 (2012)

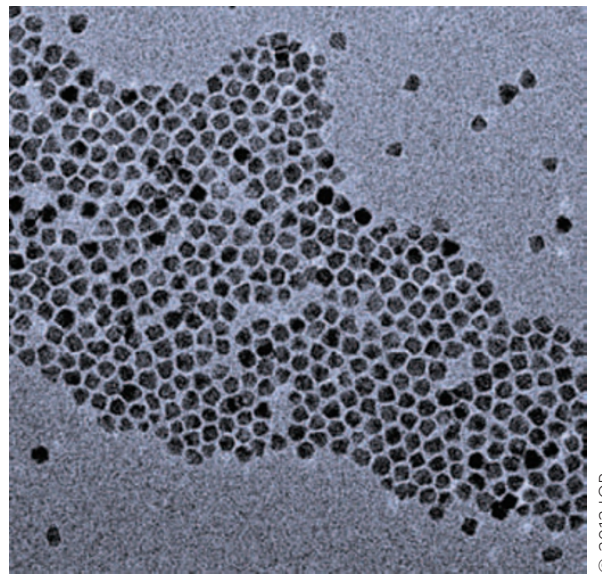

Colloidal nanocrystals are attractive nanomaterials for photonic applications such as fluorescent biological markers, single-photon sources and solar cells. Full exploitation of their properties requires the minimization of unwanted nonradiative recombination phenomena such as photoluminescence blinking. However, the exact nature of the photoexcited state in these colloidal nanocrystals is still under active debate. The recent findings of Marco Marceddu and co-workers in Italy now give a better picture of the link between photoexcited states and optical gain in CdSe-CdS core-shell nanocrystals. The researchers performed time-resolved photoluminescence measurements using a streak camera coupled to a grating spectrograph, with a temporal resolution of 25 ps and scans over a range of 2 ns. The researchers demonstrated the existence of single-, bi- and charged excitons by fitting the photoluminescence decay curves and found that Auger recombination of biexcitons was not suppressed, while optical gain and amplified spontaneous emission were from multi-exciton states and longlived charged-exciton states.

Written by James Baxter, Oliver Graydon, Noriaki Horiuchi, David Pile and Rachel Won. 